\title{
PARAMETRIC SENSITIVITY COMPARISON OF SIMULATION MODELS FOR FLYER PLATE IMPACT EXPERIMENTS
}

\author{
D. SOMASUNDARAM ${ }^{1}$, S. K. ROY ${ }^{1}$, M. TRABIA ${ }^{1}$, B. O'TOOLE ${ }^{1} \&$ R. HIXSON ${ }^{2}$ \\ ${ }^{1}$ Department of Mechanical Engineering, University of Nevada, USA. \\ ${ }^{2}$ National Security Technologies, LLC, USA.
}

\begin{abstract}
This article presents a study of the sensitivities of different parameters that affect the accuracy of simulating flyer plate impact experiments. Two approaches are explored: the CTH hydrodynamic and the LS-DYNA smoothed particle hydrodynamic (SPH) codes. Simulations using these two methods are compared to experimental data from a single-stage gas gun experiment in which a copper flyer plate impacted another copper target plate. The experiment was designed to cause spall in the target plate. The numerical simulations are conducted using these combined physics models: the Mie-Grüneisen equation of state, the Johnson-Cook compressive strength model, and spall rupture. Effects of artificial viscosity, spall strength, and computational cell size are studied and discussed with the objective of improving the accuracy of these simulations. The results are verified by applying the proposed simulation approach to other flyer plate experiments.
\end{abstract}

Keywords: CTH, Johnson-Cook material model, smoothed particle hydrodynamics, spall strength, $\mathrm{SPH}$.

\section{INTRODUCTION}

Hypervelocity meteorite and projectile impacts induce shocks and high strain rates over extremely short durations. These impacts cause materials to undergo significant elastic and plastic deformations. While there is a wealth of information describing shock physics at relatively moderate velocities, the behavior of materials under shocks associated with hypervelocities received limited attention.

The following is a brief overview of some of the recent relevant research in this area. Johnson [1] developed a model for describing spalling fracture induced by plate impact or explosives. The model was compared with copper experiments where X-radiography was used to characterize the spalling. Curran et al. [2] described spalling in terms of the microstructural changes that the material undergoes during shock. They were able to relate these changes to continuum mechanics. Molinari and Wright [3] presented a parametric study to show the effects of various parameters such as loading rate, peak loading stress, density of nucleation sites, and physical properties of the material on the spall fracture of ductile materials and the associated distribution of void sizes when a critical porosity is reached. Yu et al. [4] investigated the spall of LY12 aluminum alloy experimentally by attenuating the shock wave to produce a decaying triangular shock pulse in the samples of plate impact spall tests. Spall signals were measured using the free surface velocity of the target plates. Recently, Kanel [5] presented an experimental approach for measuring spalling under hypervelocity conditions. Escobedo et al. [6] conducted plate impact experiments to understand the effect of grain boundary distribution on the dynamic tensile response of copper. Post-impact metallographic analysis was conducted to understand the relation between spall formation and grain size. Bat'kov et al. [7] studied the evolution of damage in copper subjected to impact. Richter [8] used two computer programs, FLUKA and ANSYS AUTODYN, to model 
the physics associated with beam intercepting devices. Overall, most of this research focused on experimental understanding of the shock physics.

Simulating these experiments, however, received limited attention. Recent advancement in computational methods has provided tools that can be used for modeling these events, which can reduce the need to conduct the relatively expensive experiments. Because of the fluid-like behavior of materials under these conditions, traditional finite element methods suffer from multiple limitations when simulating these experiments where elements can be distorted significantly. Instead, various recent computer simulation codes that use a hydrodynamics concept have been developed to simulate these problems; many of them are now relatively mature.

Hypervelocity experiments are expensive to conduct. It is, therefore, important to develop an approach to simulate these experiments accurately to reduce the number of these experiments. The objective of this work is to create a model that matches the hypervelocity experimental data closely by providing recommendations for using two codes to model flyer plate impact experiments. The first code is the Lagrangian-based SPH solver in LS-DYNA [9]. The same problem was simulated using the Eulerian-based hydrocode in CTH, which was developed by Sandia National Laboratories [10]. This study can also provide some insights into the material damage incurred during impact.

\section{EXPERIMENTAL SETUP}

An experiment was conducted using a cylindrical target copper plate impacted by a flyer copper plate with the same cross-sectional surface using a single-stage gas gun to determine the fundamental dynamic properties of materials under shock conditions [11]. A Velocity Interferometer System for Any Reflector (VISAR) [12] was used as the principal diagnostic system for the experiment. The VISAR system measured the velocity of the center point on the back surface of the target plate. Both flyer and target plates remained in a one-dimensional uniaxial strain state throughout the duration of the measurement. The diameter of the plates was large enough to prevent edge release wave from perturbing the center of target free surface over the time needed to make measurements. A summary of this experiment is presented in Table 1.

Figure 1 shows a typical set of VISAR velocity results. The data exhibit four major characteristics: (1) an elastic precursor wave, (2) a plastic shock-wave rise time, (3) a plateau, and (4) a spall signature. A later drop of velocity corresponds to the formation of the spall region.

Table 1: Summary of the experiments.

\begin{tabular}{ll}
\hline & $\begin{array}{c}\text { Experiment } 1[11] \\
\text { Flyer plate material }\end{array}$ \\
\hline Flyer and target plates diameter $(\mathrm{mm})$ & $\begin{array}{l}\text { Oxygen-free high-conductivity }(\mathrm{OFHC}) \\
\text { copper }\end{array}$ \\
Flyer plate thickness $(\mathrm{mm})$ & 1.78 \\
Flyer plate velocity $(\mathrm{m} / \mathrm{s})$ & 307 \\
Target plate material & OFHC copper \\
Target plate thickness $(\mathrm{mm})$ & 4.0 \\
\hline
\end{tabular}




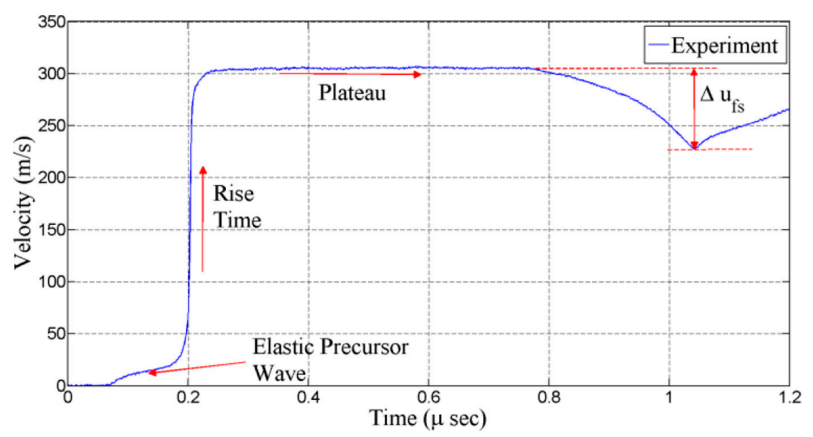

Figure 1: Typical experimental results for the measured velocity at the back surface of a one-dimensional uniaxial strain flyer plate impact [11].

In an earlier work [13], we have simulated this experiment using a Lagrangian-based SPH solver in LS-DYNA. A discussion of the variables affecting this type of simulation was presented. This work extends the ideas presented in [13] to another complementary simulation approach using the Eulerian-based hydrocode CTH. Details of the simulation results using LS-DYNA and CTH are presented and compared based on varying artificial bulk viscosity, spall strength, and zone size. The results are later verified using two additional experiments [14] to assess the robustness of the proposed approach.

\section{COMPUTATIONAL MODELS}

In this section, the effect of varying input parameters of the model is studied. Flyer plate experiments are modeled in both CTH and LS-DYNA computational software packages using the two-dimensional axisymmetric assumptions (Fig. 2). The remainder of this section describes the physics models used for simulating the experiment.

To simulate dynamic events, equations of state (EOSs) for all materials involved in impact must be chosen. A simple and robust form of EOS that is commonly used is the Mie-Grüneisen form, which is based on observing shock compression data. Mie-Grüneisen EOS can be expressed in many ways. In this work, we follow the form used in LS-DYNA [9] where pressure, $P$, is

$$
P=\frac{\rho_{0} c_{0}^{2} \mu\left(1+\left(1-\frac{\gamma_{0}}{2}\right) \mu-\frac{a}{2} \mu^{2}\right)}{\left(1-\left(S_{1}-1\right) \mu-S_{2} \frac{\mu^{2}}{\mu+1}-S_{3} \frac{\mu^{3}}{(\mu+1)^{2}}\right)^{2}}+\left(\gamma_{0}+a \mu\right) E_{I},
$$

$P$ is a function of the non-dimensionalized density change:

$$
\mu=\left(\frac{\rho}{\rho_{0}}-1\right),
$$

where $\rho$ is the current density and $\rho_{0}$ is the reference density. $S_{1}, S_{2}$, and $S_{3}$ are the coefficients of the slope of the shock velocity-particle velocity curve, $c_{0}$ is the intercept of the shock velocity-particle velocity curve, $\gamma_{0}$ is the Grüneisen coefficient, $a$ is the first-order volume 


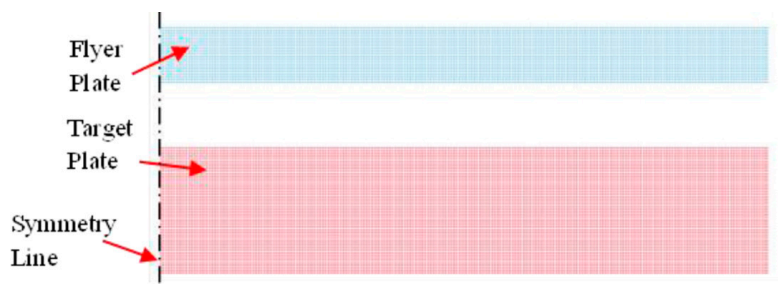

Figure 2: Typical two-dimensional axisymmetric model for flyer plate and target plate.

correction to $\gamma_{0}$, and $E_{\mathrm{I}}$ is the internal energy per unit volume. In this work, EOS parameters for the OFHC copper are based on Ref. [15]. A simplified form of the equation is used in this study by neglecting the higher order coefficients, $S_{2}$ and $S_{3}$, to reduce eqn (1) to

$$
P=\frac{\rho_{0} c_{0}^{2} \mu\left(1+\left(1-\frac{\gamma_{0}}{2}\right) \mu-\frac{a}{2} \mu^{2}\right)}{\left(1-\left(S_{1}-1\right) \mu\right)^{2}}+\left(\gamma_{0}+a \mu\right) E_{I} .
$$

Table 2 lists the parameters of the Mie-Grüneisen EOS model for copper.

The EOS does not directly contain information about material compressive strength. Therefore, it is also necessary to define a strength model for these simulations. JohnsonCook model [16] is typically used to describe mechanical performance under these impact conditions. In the Johnson-Cook material model, the flow stress is expressed as

$$
\sigma_{y}=\left(A+B\left(\varepsilon_{e f f}^{p}\right)^{n}\right)(1+C \ln (\dot{\varepsilon}))\left(1-\left(T^{*}\right)^{m}\right)
$$

where $\sigma_{y}$ is the flow stress, $A$ is the yield stress under quasi-static conditions, $B$ and $n$ are strain-hardening parameters, $m$ is the temperature sensitivity, and $C$ is the strain-rate dependence parameter. $\dot{\varepsilon}_{e f f}^{p}$ is the effective plastic strain and $\dot{\varepsilon}_{0}$ is the plastic strain rate normalized by the reference strain rate $\dot{\varepsilon}_{0}$, which can be defined as

$$
\dot{\varepsilon}=\frac{\dot{\varepsilon}_{e f f}^{p}}{\dot{\varepsilon}_{0}} .
$$

$T^{*}$ is the homologous temperature defined by the following relation:

$$
T^{*}=\frac{T-T_{r}}{T_{m}-T_{r}},
$$

where $T$ is the temperature, and $T_{\mathrm{m}}$ and $T_{\mathrm{r}}$ are the melting and reference temperatures, respectively. The parameters for this model for copper are taken from Ref. [16] and are listed in Table 3.

An accurate simulation of hypervelocity impact requires including spall rupture, which can be defined as an internal crack that is caused by tensile stress within the material that exceeds the tensile strength of the material [17]. Spall strength can be approximately calculated using the experimentally determined pull-back velocity [18], which is defined as 
Table 2: Parameters of the Mie-Grüneisen EOS model for copper.

\begin{tabular}{llll}
\hline$c_{0}(\mathrm{~m} / \mathrm{s})$ & $S_{1}$ & $\gamma_{0}$ & $\rho_{0}\left(\mathrm{~kg} / \mathrm{m}^{3}\right)$ \\
\hline 3940 & 1.489 & 1.99 & 8930 \\
\hline
\end{tabular}

Table 3: Parameters of the Johnson-Cook model for OFHC copper.

\begin{tabular}{lllllllll}
\hline$A(\mathrm{MPa})$ & $B(\mathrm{MPa})$ & $C$ & $N$ & $m$ & $\varepsilon_{0}(1 / \mathrm{s})$ & $T_{m}(\mathrm{~K})$ & $C_{v}(\mathrm{~J} / \mathrm{kg} \mathrm{k})$ & $\rho_{\text {spall }}(\mathrm{GPa})$ \\
\hline 89.7 & 292 & 0.025 & 0.31 & 1.09 & 1 & 1381 & 392 & 1.79 \\
\hline
\end{tabular}

the difference between peak-free surface velocity and the lowest free-surface velocity ahead of the spall pulse $\left(\Delta_{\mathrm{ufs}}\right)$ (Fig. 1). The spall strength can be then expressed as

$$
\sigma_{\text {spall }}=\frac{1}{2} \Delta \mu_{f s} \rho_{0} c_{b}
$$

where $c_{\mathrm{b}}$ is the bulk sound speed, which is taken as $3800 \mathrm{~m} / \mathrm{s}$ for OFHC copper. Hence, the spall strength of the OFHC copper is calculated to be $1.32 \mathrm{GPa}$ based on the results of Fig. 1 and eqn (5).

\section{IDENTIFICATION OF MODEL PARAMETERS}

Preliminary simulation results show that varying parameters of the particle approximation theory, the initial number of neighbors, and the computational method for smoothing length initialization in LS-DYNA do not affect the results significantly. On the other hand, the models show sensitivity to other varying variables such as bulk viscosity, spall strength, and mesh density. This section proposes an approach for identifying these parameters. In Sections 4.1 and 4.2, SPH particle spacing and CTH zone size are $0.01 \mathrm{~mm}$ while all CTH simulations have a zone size of $0.01 \mathrm{~mm} \times 0.01 \mathrm{~mm}$.

\subsection{Artificial bulk viscosity parameters}

Numerical simulations of material behavior under shockwave pressure often incorporate an artificial bulk viscosity parameter to compensate for shock discontinuities. In LS-DYNA, two parameters, $Q_{1}$ and $Q_{2}$, are used to define this property, where $Q_{1}$ and $Q_{2}$ are the quadratic and linear coefficients, respectively. A previous sensitivity study [13] in LS-DYNA concluded that the suitable values for $Q_{1}$ and $Q_{2}$ in SPH models were 1.5 and 1.0, respectively. Figure 3a shows the effect of varying $Q_{2}$ in the LS-DYNA model while holding all other properties constant.

A similar sensitivity study was performed with the CTH simulations. The default CTH values for the linear, BL, and quadratic, BQ, coefficients of the artificial viscosity are 0.1 and 2.0 , respectively. The model was not sensitive to changes in the shear viscosity parameter, $\mathrm{BS}$, which was left at the default value of 0.03 . Two simulations were run with BL equal to 0.1 and 1.0, respectively, while maintaining BQ at 2.0. Simulation results are shown in Fig. 3b. The results show that using the default values results in accurate simulations. While the plastic shock rise was slightly slower in both simulations than in the experiment, the CTH results are closer to the experimental data than those of LS-DYNA. 


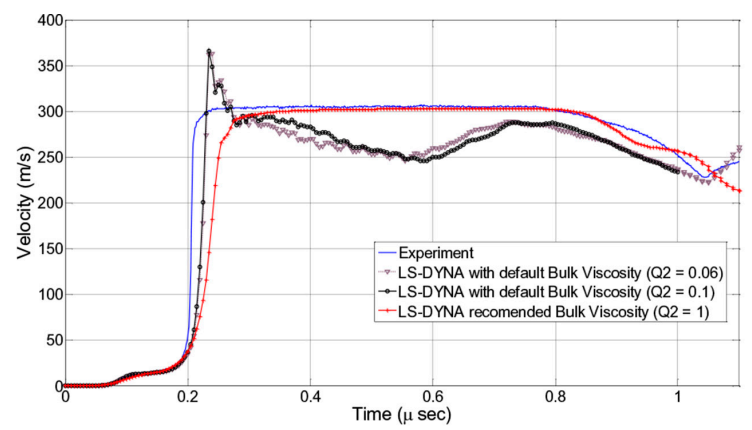

(a)

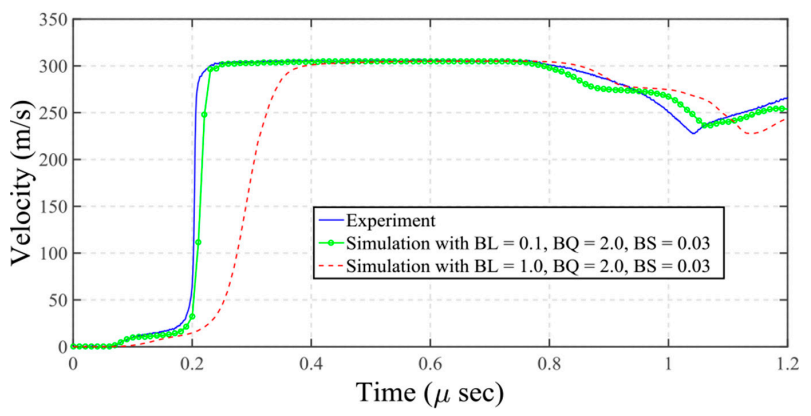

(b)

Figure 3: Effect of varying artificial bulk-viscosity parameters on (a) LS-DYNA and CTH simulations (b).

\subsection{Spall strength}

Previously, LS-DYNA simulations [13] were performed assuming a spall-strength value of $1.79 \mathrm{GPa}$ for OFHC copper. However, the results of Section 3 showed that a spall strength value of $1.32 \mathrm{GPa}$ for OFHC copper was more accurate. Therefore, LS-DYNA simulations were performed in this section using this calculated value to check the effect of spall strength (Fig. 4a). The results showed that the two simulations were practically identical for the first $1.1 \mu \mathrm{s}$. However, a spall strength value of $1.32 \mathrm{GPa}$ moved the simulated velocity closer to the corresponding feature of the experimental data, which improved the simulation's accuracy in capturing the spall signature phenomenon.

A similar study was performed with CTH hydrocode to check the effect of spall strength variation in CTH (Fig. 4b). Interestingly, CTH simulation with $1.32 \mathrm{GPa}$ showed a dip in front of the pull-back signal almost at the same time as the experimental velocity profile. However, the velocity was significantly higher afterward. Based on these results, spall strength of the OFHC copper was selected as $1.32 \mathrm{GPa}$ for both LS-DYNA and CTH simulations. This value was used in the remainder of this work.

\subsection{SPH particle spacing and CTH zone size study}

The objective of this section is to study the effect of the SPH particle spacing and CTH zone size on the flyer plate problem using the results of the two previous sections. Four models 


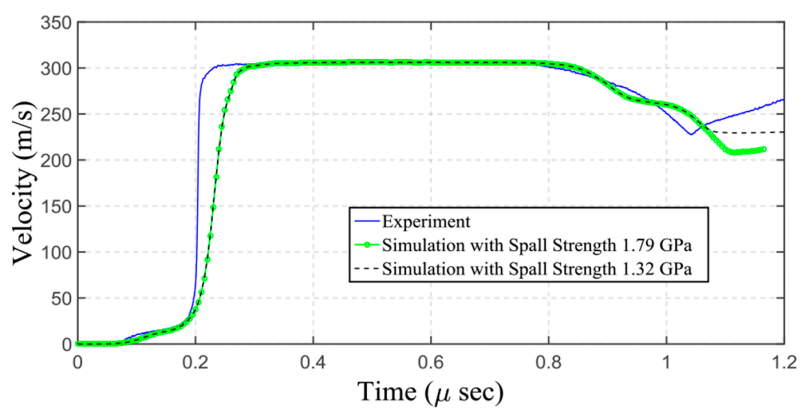

(a)

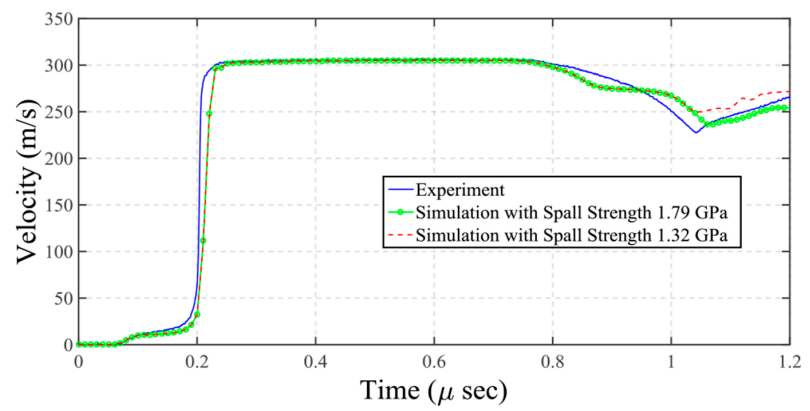

(b)

Figure 4: Effect of varying spall strength on (a) LS-DYNA and (b) CTH simulations.

with different particle spacing are created in LS-DYNA: 0.08, 0.02, 0.01, and $0.009 \mathrm{~mm}$, respectively. The results of the four cases (Fig. 5a), indicated that all cases, except for the one with the largest particle spacing $(0.08 \mathrm{~mm})$, are able to capture the elastic precursor wave and the velocity plateau. However, there was a considerable variation in the rise time of the plastic shock wave, with the $0.009 \mathrm{~mm}$ model being the most accurate. It is noted that as the particle density increases, the simulation rise time approaches the experimental curve. The problem, however, became computationally expensive, requiring approximately $23 \mathrm{~h}$ on a 46-processor server for the $0.009 \mathrm{~mm}$ particle spacing case.

A similar zone size parametric study is conducted for CTH. The results are shown in Fig. 5b. Similar to LS-DYNA, rise time becomes shorter as smaller zones are used. Both the 0.01 and $0.02 \mathrm{~mm}$ zones capture the elastic and plastic waves, and reach the same plateau value. However, these simulations exhibit ringing structure after the spall signature. Interestingly, the $0.08 \mathrm{~mm}$ zone calculation seems to capture the expected ringing signature best.

\section{VERIFICATION}

The robustness of the results of the previous section are verified using the two sapphirecopper experiments of Table 4 where sapphire flyer plates impacted stationary copper plates at velocities different from what was used in the previous section (401 and $142 \mathrm{~m} / \mathrm{s}$ versus $307 \mathrm{~m} / \mathrm{s})$. 


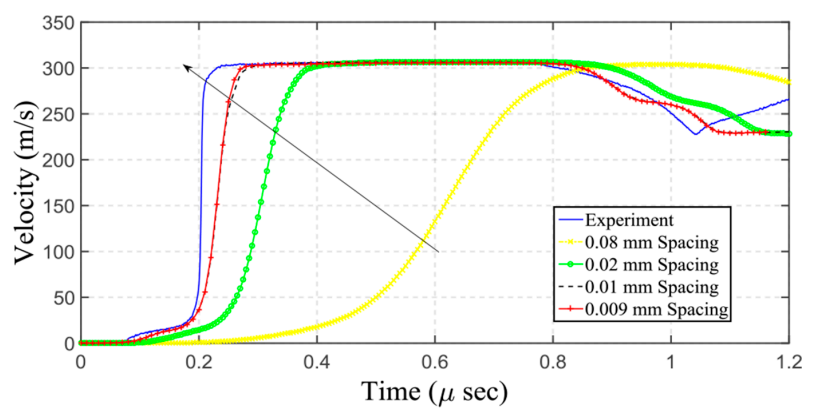

(a)

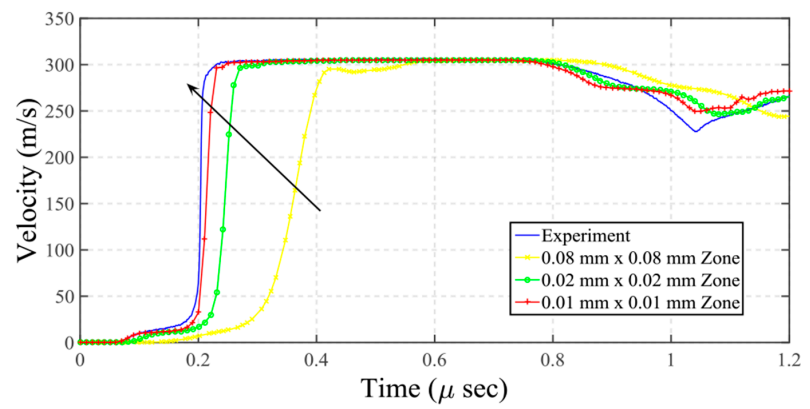

(b)

Figure 5: Effect of particle density on (a) LS-DYNA and (b) CTH simulations.

Table 4: Summary of the verification experiments.

\begin{tabular}{lll}
\hline & Experiment 2 [14] & Experiment 3 [14] \\
\hline Flyer plate material & Sapphire & Sapphire \\
Flyer and target plates diameter $(\mathrm{mm})$ & 15 & 15 \\
Flyer plate thickness $(\mathrm{mm})$ & 3.053 & 2.979 \\
Flyer plate velocity $(\mathrm{m} / \mathrm{s})$ & 401 & 142 \\
Target plate material & OFHC copper & OFHC copper \\
Target plate Thickness $(\mathrm{mm})$ & 4.246 & 4.563 \\
\hline
\end{tabular}

The parameters of the Mie-Grüneisen EOS and Johnson-Cook material model for copper were maintained as listed in Section 3. Similarly, the final values of the artificial bulk viscosity parameters and particle and zone spacing of Section 4 were used in the simulations of this section.

For these two sapphire-copper impact experiments, it was decided to use an isotropic elastic plastic material model for the sapphire as these experiments were designed such that the stress in the sapphire plates is kept below sapphire's yield strength during the impact. The use of an isotropic material in the case of the anisotropic sapphire material can be justified by the one-dimensional nature of stress and since all the experiments were performed below the yield point of sapphire. The values of the material model parameters are presented in 
Table 5 [12]. $E$ is the modulus of elasticity, $G$ is the shear modulus, $K$ is the bulk modulus, $S_{\mathrm{Y}}$ is the yield strength, and $E_{\tan }$ is the slope of the stress-strain curve in the plastic phase. These values are based on Ref. [19] except for $S_{\mathrm{Y}}$ and $E_{\tan }$, where the values listed in [19] could not be used as they were based on the characteristics of a single crystal.

Since the experiments were designed such that the stress in the sapphire plates was kept below the sapphire's yield strength, the sapphire plates did not spall. Therefore, there was no need to identify the sapphire's spall strength.

In CTH, the Mie-Grüneisen EOS model for sapphire was based on the data of [12] for the case when stresses are below yielding. The parameters of this model are listed in Table 6 . LS-DYNA, however, does not allow incorporation of EOSs with the material card that was used (*MAT_ISOTROPIC_ELASTIC_PLASTIC). In this case, a simpler model was used where the shock wave speed is equal to $\sqrt{E / \rho}$.

Figures 6 and 7 show the results of simulating Experiments 2 and 3, respectively, using LS-DYNA and CTH software packages. The simulation results are in general consistent with the conclusions in Section 4. Both LS-DYNA and CTH were able to simulate the elastic precursor, especially in Experiment 2 (higher velocity). CTH showed to be more robust in simulating both experiments accurately and capturing rise time, plateau, and spall signature. It is noted that LS-DYNA consistently produces longer rise time, which may be attributed to the failure to incorporate the EOSs of the sapphire in the model. Smaller particle spacing and zone size may result in improved simulation results. However, a combination of hardware and software limitations forced us to stop at these values.

Table 5: Parameters of the isotropic elastic plastic material model for sapphire.

\begin{tabular}{lllll}
\hline$E(\mathrm{GPa})$ & $G(\mathrm{GPa})$ & $K(\mathrm{GPa})$ & $S_{\mathrm{Y}}(\mathrm{MPa})[11]$ & $E_{\tan }(\mathrm{GPa})[11]$ \\
\hline 345 & 145 & 250 & 190 & 18 \\
\hline
\end{tabular}

Table 6: Parameters of the Mie-Grüneisen equation of state model for sapphire.

\begin{tabular}{llll}
\hline$c_{0}(\mathrm{~m} / \mathrm{s})$ & $S_{1}$ & $\gamma_{0}$ & $\rho_{0}\left(\mathrm{~kg} / \mathrm{m}^{3}\right)$ \\
\hline 11,190 & 1.0 & 1.50 & 3985 \\
\hline
\end{tabular}

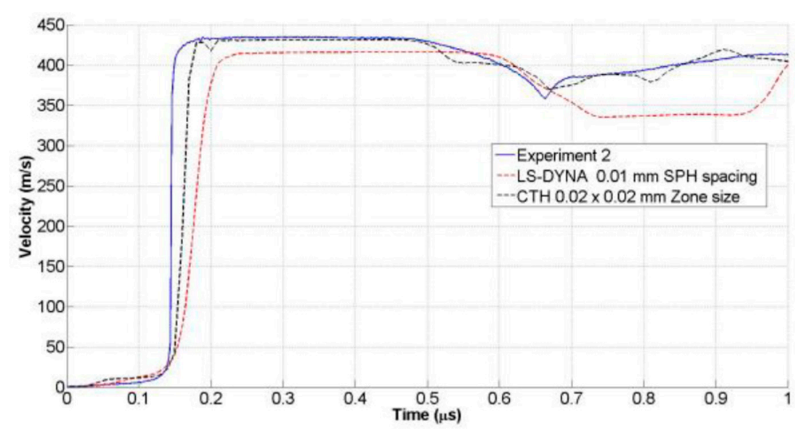

Figure 6: Results of simulating Experiment 2 using the recommended parameters for LS-DYNA and CTH software packages. 


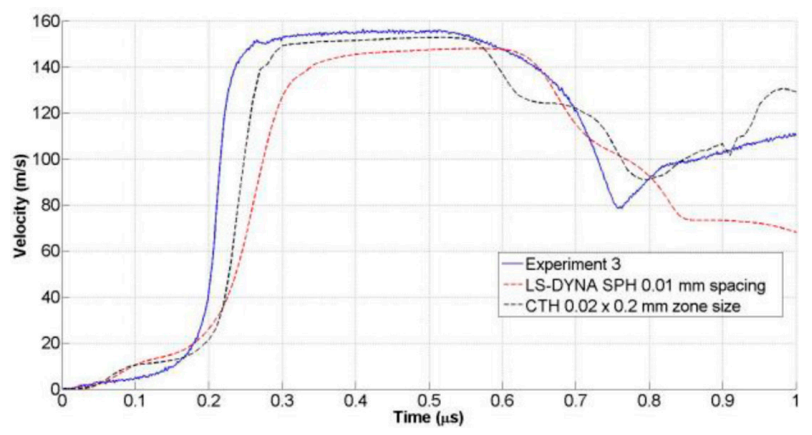

Figure 7: Results of simulating Experiment 3 using the recommended parameters for LS-DYNA and CTH software packages.

\section{CONCLUSIONS}

This study is an effort to develop an approach for accurately simulating flyer plate impact experiments using the CTH hydrocode and SPH technique in LS-DYNA. Using an OFHC copper-OFHC copper flyer plate experiment, it was shown that the sensitivities of both models depend on several parameters. The results indicate that the selection of the appropriate bulk viscosity parameters will lead to a numerically stable simulation in both modeling approaches. Also, it is recommended to use a spall-strength value that is based on the experimental data. The results show that increasing the particle density in SPH and using finer zone size in CTH improve the accuracy of capturing the elastic and plastic waves. Additional work is still needed to capture the spall signature accurately. It is noted that while the results improve by using closer particles or zones in LS-DYNA and CTH, simulation time significantly increases. Therefore, a tradeoff between accuracy and computational time should be considered. These results were confirmed when simulations of two additional sapphire-OFHC copper flyer plate experiments were conducted.

Comparing the results showed that LS-DYNA and CTH are useful in simulating flyer plates experiments. The results also indicated that $\mathrm{CTH}$ is a more robust tool that produces more accurate simulations under various conditions. Additional experiments may help improving the accuracy of these simulations. Also, added improvement of material models and increase of computational power will help reduce the need of conducting expensive experiments.

\section{ACKNOWLEDGEMENT}

This manuscript has been authored by National Security Technologies, LLC, under contract no. DE-AC52-06NA25946 with the U.S. Department of Energy and supported by the SiteDirected Research and Development Program. The United States Government retains and the publisher by accepting the article for publication acknowledges that the United States Government retains a non-exclusive, paid-up, irrevocable, worldwide license to publish or reproduce the published form of this manuscript, or allow others to do so, for United States Government purposes.

\section{REFERENCES}

[1] Johnson, J. N., Dynamic fracture and spallation in ductile solids. Journal of Applied Physics, 52(4), pp. 2812-2825, 1981. doi: http://dx.doi.org/10.1063/1.329011

[2] Curran D. R., Seaman, L., \& Shockey, D. A. Dynamic failure of solids. Physics Reports, 147(5-6), pp. 253-388, 1987. doi: http://dx.doi.org/10.1016/0370-1573(87)90049-4 
[3] Molinari, A. \& Wright, T. W., A physical model for nucleation and early growth of voids in ductile materials under dynamic loading. Journal of the Mechanics and Physics of Solids, 53(7), pp. 1476-1504, 2005. doi: http://dx.doi.org/10.1016/j.jmps.2005.02.010

[4] Yu, Y., Chen, D., Tan, H., Wang, H., Xie, S. \& Zhang, M., Spall investigations for LY12 Al using triangular waves. International Journal of Impact Engineering, 34(3), pp. 395-404, 2007. doi: http://dx.doi.org/10.1016/j.ijimpeng.2005.11.006

[5] Kanel, G. I., Spall fracture: methodological aspects, mechanisms, and governing factors. International Journal of Fracture, 163(1-2), pp. 173-191, 2010. doi: http:// dx.doi.org/10.1007/s10704-009-9438-0

[6] Escobedo, J. P., Dennis-Koller, D., Cerreta, E. K., Patterson, B. M., Bronkhorst, C. A., Hansen, B. L. \& Lebensohn, R. A., Effects of grain size and boundary structure on the dynamic tensile response of copper. Journal of Applied Physics, 110(3), 033513, 2011. doi: http://dx.doi.org/10.1063/1.3607294

[7] Bat'kov, Y. V., Ignatova, O. N., Kondrokhina, I. N., Malyshev, A. N., Nadezhin, S. S., Podurets, A. M. \& and Preston, D. L., Specific features of the damage nucleation stage under severe loading of copper. Physics of the Solid State, 53(4), pp. 768-772, 2011. doi: http://dx.doi.org/10.1134/S1063783411040068

[8] Richter, H., Simulating transient effects of pulsed beams on beam intercepting devices, Doctoral dissertation, CERN, 2011.

[9] Hallquist, J. O., LS-DYNA Keyword User's Manual, Version 971, 1, Livermore Software and Technology Corporation: Livermore, CA, 2008.

[10] Hertel, Jr., E. S., CTH: a software family for multi-dimensional shock physics analysis, ShockWaves @ Marseille I, Springer: Berlin, Heidelberg, pp. 377-382, 1995.

[11] Hixson, R. S., Private communication, 9 September 2013, National Security Technologies, LLC: USA.

[12] Barker, L. M. \& Hollenbach, R. E., Shock-wave studies of PMMA, fused silica, and sapphire. Journal of Applied Physics, 41(10), pp. 4208-4226, 1970.

[13] Somasundaram, D., Trabia, M., O'Toole, B., \& Hixson, R., Variables affecting smoothed particle hydrodynamics simulation of high velocity flyer plate impact experiments. WIT Transactions on Structures Under Shock and Impact XIII, WIT Press, ISSN 174-4498, 2014.

[14] Hixson, R. S., Gray, G. T., Rigg, P. A., Addessio, L. B., \& Yablinsky, C. A., Dynamic damage investigations using triangular waves, Shock Compression of Condensed Matter-2003: Proceedings of the Conference of the American Physical Society Topical Group on Shock Compression of Condensed Matter, 706 (1), 2004.

[15] Marsh, S. P., LANL Shock Hugoniot Data, University of California Press: Oakland, CA 1980.

[16] Johnson, G. R. \& Cook, W. H., A constitutive model and data for metals subjected to large strains, high strain rates and high temperature, Proc. of 7th International Symposium on Ballistics, The Hague, The Netherlands, pp. 541-547, 1983.

[17] Grady, D., The spall strength of condensed matter. Journal of the Mechanics and Physics of Solids, 36(3), pp. 353-384, 1988. doi: http://dx.doi.org/10.1016/00225096(88)90015-4

[18] Mukherjee, D., Ray, A., Sur, A., Joshi, K. \& Gupta, S., Shock induced spall fracture in polycrystalline copper. AIP Conference Proceedings, 1591, pp. 608, 2014. doi: http:// dx.doi.org/10.1063/1.4872691

[19] Sapphire Properties, http://www.roditi.com/SingleCrystal/Sapphire/Properties.html 